Tohoku J. Exp. Med., 1992, 168, 459-466

\title{
Anatomical Study of Preaxial Polydactyly in 158 Hands
}

\author{
Sayeedul Islam, Ichiro Oka and Shinya Fujita \\ Department of Plastic and Reconstructive Surgery, Tohoku \\ University School of Medicine, Sendai 980
}

\begin{abstract}
Islam, S., Oka, I. and Fujtta, S. Anatomical Study of Preaxial Polydactyly in 158 Hands. Tohoku J. Exp. Med., 1992, 168 (3), 459-466 — Preaxial polydactyly is a common congenital hand anomaly. Here in 138 patients with 158 duplicated thumbs, a new classification of this anomaly was done from analysis of radiographs and operation records. Initially all the duplicated thumbs were arranged into from Type I to VII on the basis of radiological extent of bifurcation. Appendage type was noted as a separate entity. Type IV was found the most common and had 53 thumbs $(33.6 \%)$ involved. But findings on surgery indicated that the exact level of bifurcation varied in each type, and Type VII became three times as frequent as previously noted from x-ray examination at the time of surgery. Radiologically encountered duplications at IP (Type II), MP (Type IV) and CM (Type VI) joints were specifically classified into five groups from A to $\mathrm{E}$ on the basis of the structural changes detected on surgery. Details in each group were discussed.—congenital anomaly; thumb polydactyly ; classification
\end{abstract}

Preaxial polydactyly is the most common of congenital hand anomalies, found in this subcontinent (Leung et al. 1982; Oka et al. 1988). Soon after birth, parents seek surgical treatment, and mostly surgery is carried out within twelve months depending on the clinical types. Several classfications were proposed (Wassel 1969; Akiyama 1986), among which Wassel's classification (1969) is being widely used in clinical fields. Interestingly sometimes clinically recognized types do not correspond with the findings recorded at surgery. Reason behind it is the presence of cartilaginous epiphysis which does not show bifurcation level between two duplicated components in immature hand. So the classifications become inappropriate to some extent. It has been understood that radiological classification alone has little value in detection of clinical types as well as in preoperative assessment.

To encounter discrimination between radiological type and surgically detected criteria, an appropriate classification is designed, which will be more effective and clinically applicable in treatment of polydactylic patients.

\section{Material and Method}

In the period of 10 years from 1980 to 1989 , the total 138 patients with 158 duplicated

Received March 5, 1991 ; revision accepted for publication October 1, 1992. 

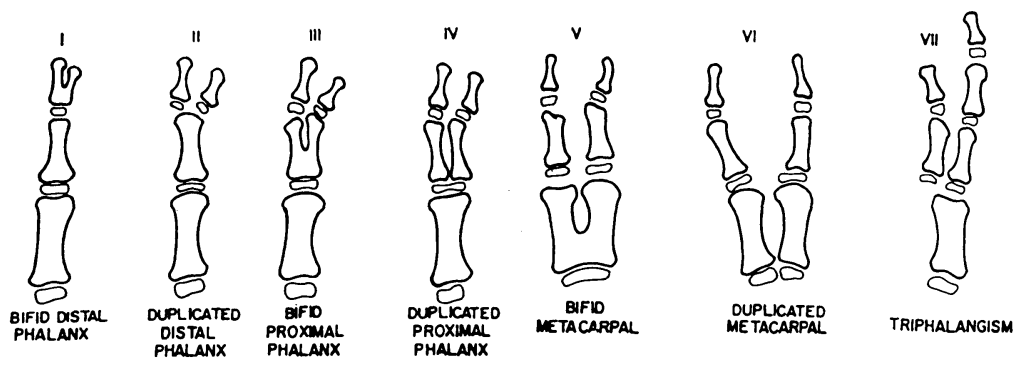

Fig. 1. Seven types of thumb polydactyly distinguished after review of 70 cases of radial polydactyly. (Wassel 1969)

thumbs underwent surgical treatment and subsequent follow-up in Department of Plastic and Reconstructive Surgery, Tohoku University School of Medicine. Out of 138 patients 72 were male and 66 were female. All these patients had detailed operation records and radiographs sufficient for analysis.

The thumbs involved were as follows : right in 55 patients ; left, 63 ; and bilateral, 20 . The age at surgery ranged from 6 months to 14 months, and the average was 12 months. Surgery for duplicated thumbs of the appendage type was carried out within 3 months after birth unlike that for well-formed stable duplicated thumbs.

The duplicated thumbs in this series were initially arranged into eight types (Type I to Type VII according to Wassel's classification and the appendage type) on the basis of their roentgenograms at the time of operation (Fig. 1). Next, the operative records were analyzed, the resected digits were macroscopically or microscopically examined, and the findings were compared with the preoperative roentgenograms.

\section{RESUlts}

Of this series the most common form in the preoperative roentgenograms was Type IV, which had $53(33.6 \%)$ thumbs involved, followed by Type II, which had $36(22.8 \%)$ thumbs. The rest of them, according to number, were the appendage type and Types VI, VII, V, III and I (Table 1). The appendage type, not

TABLE 1. Number of thumbs according to Wassel's classification

\begin{tabular}{ccc}
\hline Type & Number of thumbs & Percentage \\
\hline I & 6 & 3.8 \\
II & 36 & 22.8 \\
III & 8 & 5.1 \\
IV & 53 & 33.6 \\
V & 10 & 6.3 \\
VI & 11 & 6.9 \\
VII & 11 & 6.9 \\
Appendage & 23 & 14.6 \\
& & \\
Total & 158 & 100 \\
\hline
\end{tabular}



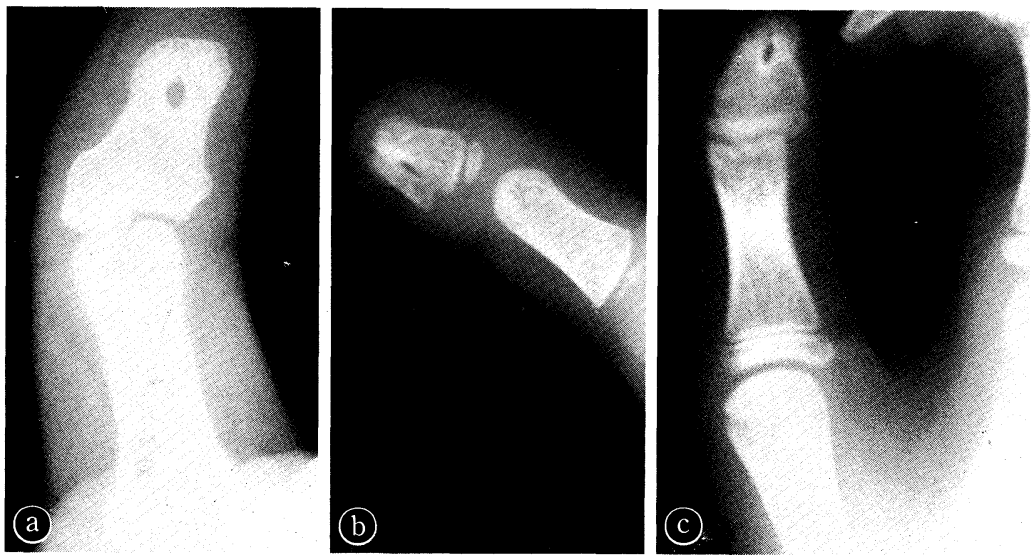

Fig. 2. Peculiar presentation in Type I. a) Fenestration in the distal phalanx. b) Both distal phalanges are attached at the head and base. c) Lipping at the tip.

mentioned by Wassel, was recorded in $23(14.6 \%)$ thumbs.

Six thumbs which had a peculiar deformity in the distal phalanx like oval fenestration in its distal third (Fig. 2a), distal phalanges fused at the bases and tips (Fig. 2b), and lipping at the tip (Fig. 2c) were grouped as Type I. Clinically these thumbs had a single nail with broad thumb tip. They were detected in routine radiological investigation.

In Type III and Type V no special feature was detected other than previously mentioned criteria (Fig. 1). Type III was the least common and contained 8 $(5.1 \%)$ duplicated thumbs only. Type V had $10(6.3 \%)$ duplicated thumbs and their bifurcations were through metacarpals.

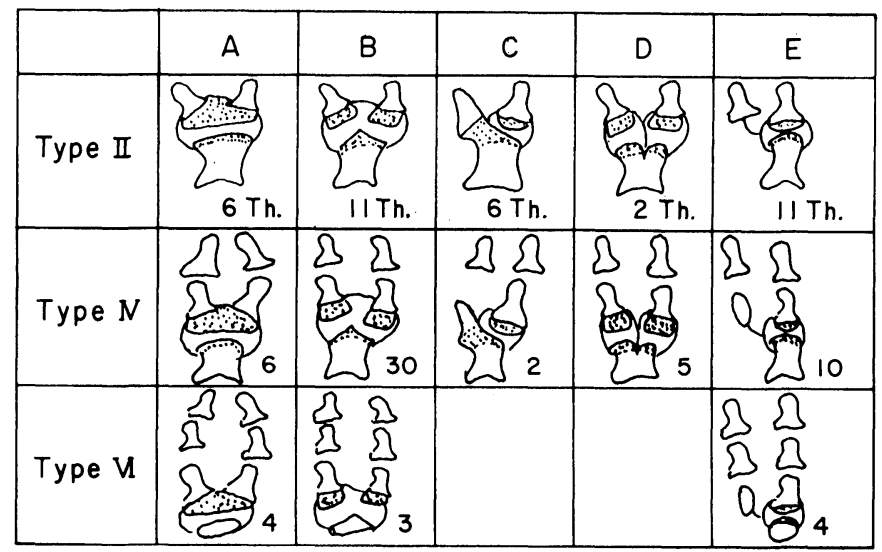

Fig. 3. Illustration of five groups in duplication at IP (Type II), MP (Type IV) and CM (Type VI) joints with their number of thumbs involved. Dotted areas represent cartilaginous parts. 


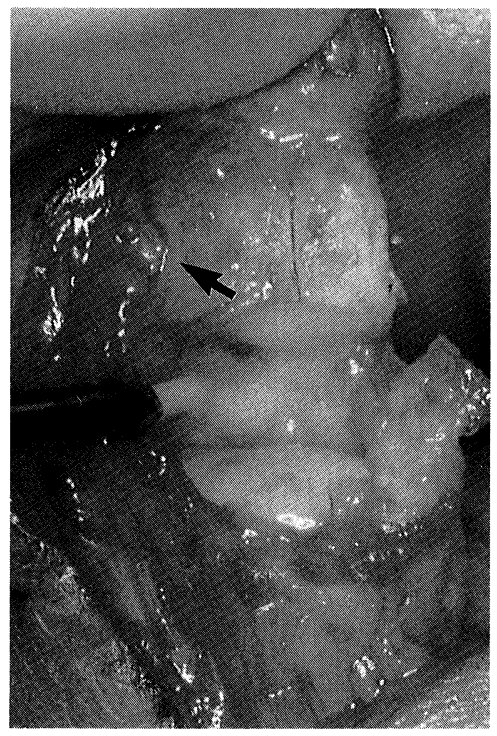

Fig. 4. Type II A shows broad proximal phalanx head but articular surface is smooth and unaltered. Distal phalanx bases are attached by cartilage (arrow).

Radiologically detected duplication at the joint, i.e. duplication at interphalangeal (IP) (Type II), metacarpophalangeal (MP) (Type IV) and carpometacarpal (CM) joints (Type VI), was found to be contradictoy to structures detected at surgery. They were hence classified into five groups depending on the structural findings at surgery (Fig. 3).

Group A. Radiological duplication level was at the joint, but at surgery the phalanx or metacarpal bases were found united each other by cartilage (Fig. 3A). The duplicated phalanges or metacarpals diverged from each other and had a broad proximal component head with no alteration in joint structure (Fig. 4). Six thumbs in Type II, 6 thumbs in Type IV and 4 thumbs in Type VI were classified as Group A.

Group B. Here bifurcation divided cartilagious epiphysis at the duplicated phalanx or metacarpal bases and extended into the joint. Proximal joint component head had two articular surfaces separated by elevated cartilagious ridge. All together there had four articular components but the joint was mutual (Fig. 3B). Radiologically duplicated members were less diverging from each other. This group included 11 thumbs in Type II, 30 thumbs in Type IV and 3 thumbs in Type VI.

Group $C$. In this group radiological duplication occured at the joint, but its unilateral member (mainly radial member) was not involved in the joint formation and remained attached by its base cartilage to the proximal component (Fig. 3C). Six thumbs in Type II and 2 thumbs in Type IV had these changes.

Group D. Radiological duplication level was at the joint, but actual bifurca- 
tion deepened into the osteocartilaginous portion of the proximal component head and, forming two heads to articulate with corresponding duplicated members (Fig. 3D). Two separate joints were formed with their own joint structures. Two thumbs in Type II and 5 thumbs in Type IV had these changes. Radiologically a single broad proximal component head was noted and duplicated members were mostly parallel.

Group $E$. One of the two component members of duplicated thumb did not participate in the joint formation and remained outside the joint, being attached to the joint capsule by fibrous band. Radiologically this member was hypoplastic (Fig. 3E). Eleven thumbs in Type II, 10 thumbs in Type IV, and 4 thumbs (2 radial and 2 ulnar members) in Type VI were found attached by fibrous tissue.

Appendage type appeared as hypoplastic extra digit on the radial surface of the thumb at any level. Its base was unstable or floating, without ligamentous or

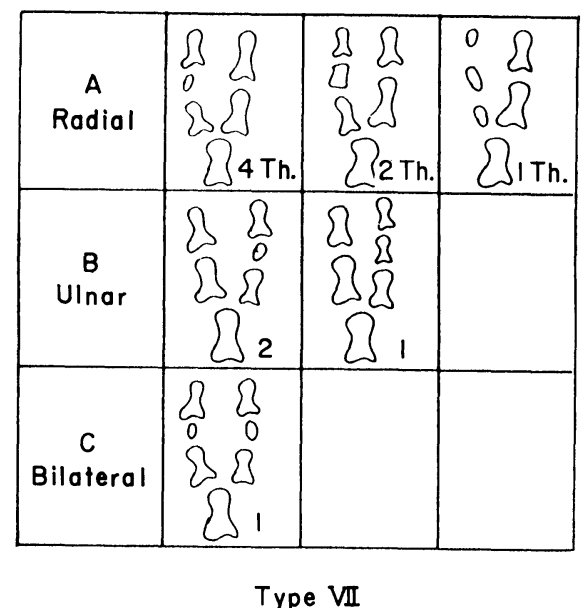

Fig. 5. Illustration of radiologically presented type VII, involving radial, ulnar and bilateral members. The number of thumbs involved are indicated.

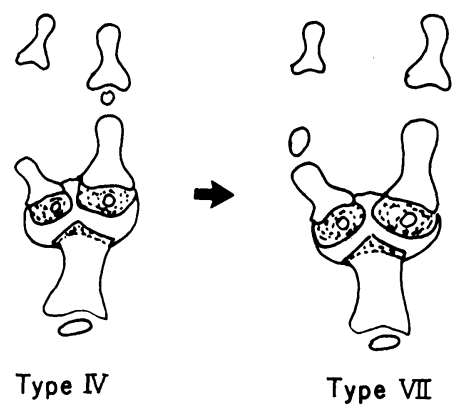

Fig. 6. Type IV shows elongated area between two phalanges, and Type VII had osseous element in that elongated area. 
a

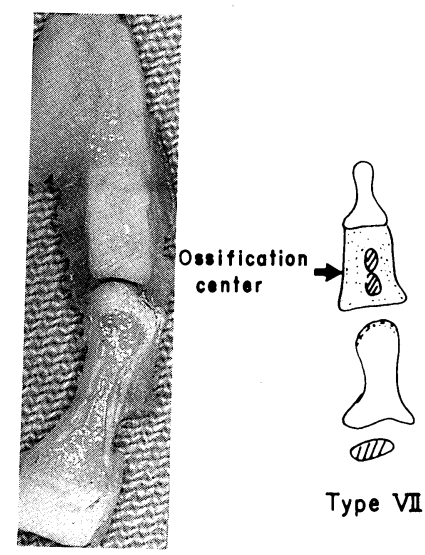

b

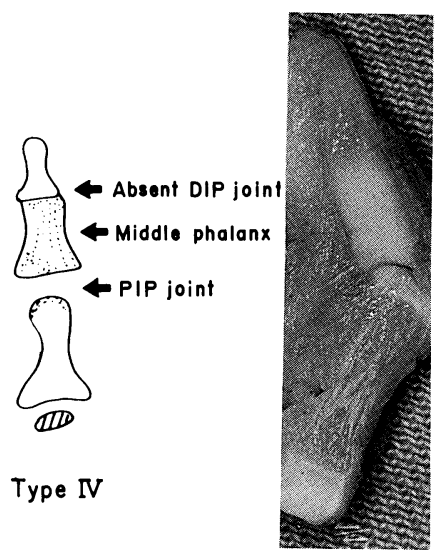

Fig. 7. Longitudinal sections of Type VII and Type IV. a) Type VII shows a long cartilaginous area proximal to distal bones; two ossification areas are seen in it. This area is supposed to be a middle phalanx uniting with a distal bone. b) Type IV has a cartilaginous middle phalanx.

bony structure. This type was not mentioned by Wassel, but designated here as appendage type, which contained 23 thumbs (14.6\%) out of 158 duplicates (Table $1)$.

Type VII means a triphalangeal thumb or elements of triphalangeal thumbs accompanied by a normal thumbs, according to Wassel. Here both well-formed bones and osseous elements were noted in the middle phalanx. Out of 158 duplicated thumbs, 11 contained osseous element or well-formed bones in either radial, ulnar or both members (Fig. 5).

In Type IV, 23 out of the 53 duplicates were radiologically shown to have an elongated area between the proximal and distal phalanges of either of the members. These 23 thumbs were different from Type VII only in having no osseous element in the elongated area (Fig. 6). Longitudinal section of excised component of both Type IV and VII had a long cartilagious area proximal to the distal bone and one or two ossification centers in it (Fig. 7).

\section{Discussion}

Preaxial polydactyly means a duplication of the first digital ray. Appropriate classification is essential to recognize clinical types. Classification was done on radiological and operative findings previously (Akiyama 1986). The appendage type mentioned here was previously described by one of the co-authors (Fujita 1982). Here a new and systematic classification is designed depending both on operative findings and radiological features. In this series duplications at IP, MP, or CM joints were classified into five groups and clinical differences between each group were noted. In Group A, both the duplicated components moved 
together, and almost full passive movement was noted at the joint. There was no side-to-side movement between the duplicates due to cartilage attachment at their bases (Fig. 3A).

In Group B, clinically passive movement was restricted due to two different angled articular surfaces at the proximal head of the joint (Fig. 3B); at operation it was required to make a congruent proximal component of the joint by shaving a central eleveted cartilaginous portion. There was passive side-to-side movement at the duplicated bases, which was noted as an identification point in this group.

In Group C, there was cartilage attachment to the proximal bone in the unilateral member, and clinically there was no passive movement at the joint (Fig. 3C). There was no volar skin crease. These changes were detected in duplicated thumbs of Type II and Type IV only. In this group reconstruction of the proximal joint head was difficult, but appropriate surgery might be offered if they are recongnized prior to surgery.

In Group D, two separete joint were detected at the duplicated bases. Unlike Group B, there was depression at the proximal component head of the joint (Fig. 3D). Clinically this group was difficult to differentiate from group B, because of its side-to-side movement at the joint similar to that of group B.

In group $\mathrm{E}$ it was characterized by its fibrous attachment of the unilateral component base to the joint capsule. In most cases this member was hypoplastic radiologically.

Here all these five groups are the newly recognized types, designed from operation records and radiological analyses. In Types II, IV, and VI, duplicated thumbs were arranged into five groups, but if they are left untreated, their types would be changed; six thumbs in Type II-A (17\% of 36 thumbs in Type II) would migrate to Type I, 6 thumbs in Type IV-A (11\% of the 53 in Type IV) to Type III, and 4 thumbs in Type VI-A ( $36 \%$ of the 11 in Type VI) to Type V. In

TABLE 2. Number of thumbs according to operative findings

\begin{tabular}{ccc}
\hline Type & Number of thumbs & Percentage \\
\hline I & 12 & 7.6 \\
II & 30 & 19.0 \\
III & 14 & 8.8 \\
IV & 25 & 15.8 \\
V & 14 & 8.8 \\
VI & 7 & 4.4 \\
VII & 33 & 21.0 \\
Appendage & 23 & 14.6 \\
& & \\
Total & 158 & 100 \\
\hline
\end{tabular}


Type IV $23(42 \%)$ thumbs had an elongated area between the proximal and distal phalanges; this cartilaginous area was supposed to be the middle phalanx uniting with distal bone without DIP joint formation and would be converted to Type VII (Figs. 6 and 7). When corrected on the basis of operative findnigs, the radiologically noted incidence in Type IV became half and that of Type VII became three times higher. The 158 duplicated thumbs, which were detected radiologically, were reclassified according to operative findings, and the rate of occurrence was calculated as follows : $33(21 \%)$ thumbs in Type VII was the highest, next to it was $30(19 \%)$ thumbs in Type II, followed by $25(15.8 \%)$ thumbs in Type IV, $23(14.6 \%)$ thumbs in appendage type, $14(8.8 \%)$ thumbs in Type III, $14(8.8 \%)$ thumbs in Type V, $12(7.6 \%)$ thumbs in Type I, and $7(4.4 \%)$ thumbs in Type VI (Table 2). The comparative record proved that occurence rate of Type VII is higher than that of Type IV in polydactylic hands.

\section{References}

1) Akiyama, M. (1986) The patterns of duplication and the forms of the duplicated digits in thumb polydactyly. J. Jpn. PRS., 6, 377-389. (in Japanese)

2) Fujita, S. (1982) Treatment of polydactyly of the thumb. J. Jpn. PRS., 2, 200-213. (in Japanese)

3) Leung, P.C., Chan, K.M. \& Cheng, J.Y.C. (1982) Congenital anomalies of the upper limb among the Chinese population in Hong Kong. J. Hand Surg., 7, 563-565.

4) Oka, I., Watanabe, H., Nagumo, M. \& Fujita, S. (1988) Incidence of congenital anomalies in hand. J. Jpn. Soc. Surg. Hand., 5, 771-774. (in Japanese)

5) Wassel, H.D. (1969) The results of surgery for polydactyly of the thumb. Clin. Orthop., 64, 175-193. 